\title{
Cardiac Hydatid Cyst Rupture and Embolization Causing Lower Limb Ischemia and Multiple Infarcts: A Case Report
}

Malik Azhar Hussain ${ }^{1 *}$, Ramadan Alatawneh ${ }^{2}$, Muhammad Omer Afzal Bhatti ${ }^{3}$, Mohammad Akram Randhawa ${ }^{4}$, Adnan Anwar $^{5}$ and Neeta Maheshwary ${ }^{6}$

${ }^{1}$ Department of General Surgery, College of Medicine, Northern Border University Arar, Kingdom of Saudi Arabia

${ }^{2}$ Department of Vascular surgery, Arar Central Hospital Arar, Northern border, Kingdom of Saudi Arabia

${ }^{3}$ Department of Radiology, College of Medicine, Northern Border University Arar, Kingdom of Saudi Arabia

${ }^{4}$ Department of Pharmacology, College of Medicine, Northern Border University, Arar, Saudi Arabia

${ }^{5}$ Department of Physiology, Altibri Medical college, Pakistan

${ }^{6}$ Manager Medical Affairs and Clinical Research, Hilton Pharma, Pakistan

Received: 26 October, 2017; Accepted: 29 November, 2017; Published: 04 December, 2017

*Corresponding author: Malik Azhar Hussain, Department of Surgery, College of Medicine, Northern Border University, Arar, Saudi Arabia, Tel: 00966503161516; E-mail: drmalikazhar@yahoo.com

\begin{abstract}
Hydatid Cyst (HC) is a complex, chronic parasitic disease with a world-wide distribution. It is endemic in communities where infected meat of grazing animals is consumed by the humans. Those grazing animals acquire parasitic infection by eating grass that is contaminated with dog fecal matter, which contains eggs of parasite that develop into hydatid cyst. HC is caused by the larval stage (daughter cells/ brood capsules) of Echinococcus granulosus found in intermediate host of sheep and human beings. Dog contains adult form of parasite in intestine and is the true host. HC diagnosis is based on serological reactions like Casoni test and ELISA for Echinococcus multilocularis and serves as adjunctive role along with radiological investigations. The diagnosis of cardiac HC is done by echocardiography, X-ray chest, contrast spiral computerized tomography, and/or magnetic resonance imaging. Cardiac hydatid cyst rupture from left heart causing ischemia and distal infarction by multiple emboli is a rare entity. We report such a case of a 24 year young Saudi male who died of Systemic Inflammatory Response Syndrome and Multiple Organ Dysfunction Syndrome, while under treatment for bilateral lower limbs ischemia.
\end{abstract}

Keywords: Ruptured Cardiac HC; Embolism; Lower Limb Vascular Ischemia and Infarction; Systemic Inflammatory Response Syndrome; Multiple Organ Dysfunction Syndrome

\section{Introduction / Literature Review}

Cardiac Hydatid Cyst (HC) is a rare parasitic infection transferred to humans by eating raw or uncooked meat of grazing animals, such as sheep, which acquires parasitic infection by eating grass contaminated with dog's fecal matter. Upon eating grass the sheep develop hydatid cyst in their body. Lifethreatening pathology starts in humans after eating infected sheep-meat. Upon ingestion by humans the HC lodges in the liver via portal circulation, then to right heart, pulmonary artery, lungs and left heart. $75 \%$ of HCs are seen in the liver, $15 \%$ in the lungs and $10 \%$ in the left side of heart [1-6].
HC parasitic disease is mostly seen in the South America, the Mediterranean belt, Eastern Europe, Gulf and Middle East, Eastern Africa, Central Asia, China, and Russia [1-3]. Cardiac hydatid disease is a rare, but life-threatening disease. It has fatal cardiac complications such as valvular dysfunction, cardiac wall rupture, distal embolization, anaphylactic reactions, atrial and ventricular conduction defects and sometime congestive heart failure. The most common cardiac location of $\mathrm{HC}$ is the ventricular myocardium followed by sub-epicardium and coronary arteries, which may lead to acute sudden myocardial infarct without prior history of heart disease. HC rarely ruptures in the pericardial space causing cardiac tamponade. The left ventricle is damaged twofold to threefold more frequently than the right ventricle. The most dangerous complication of HC is the cyst rupture. After cyst rupture two third of the patients die from anaphylaxis/septic shock or embolism related complications [1-6].

For the diagnosis of cardiac HC echocardiography, X-ray chest, contrast spiral computerized tomography, and/or magnetic resonance imaging is usually employed [2, 3]. Its confirmation as tinea echinococcus cyst is done by serological reactions, such as Casoni test or Elisa [1].

The side effects of HC could be jaundice, cardiac asthma, or anaphylactic shock that can be fatal [4,5]. Rarely, HC in the left side of heart may rupture and embolize to cause ischemia of distant organs [6]. Hydatid cyst arterial embolism cannot be immediately distinguished from other causes of thromboembolism from left heart $[7,8]$.

Medical treatment is mandatory by chemotherapy to kill the parasitic HC cysts and to avoid their re-implantation (seedling) in other organs. Surgical intervention is needed for complications, related to obstruction of adjacent blood vessels or ducts, or related to embolization causing ischemia or infarcts $[8,9]$. 


\section{Case Presentation}

Cardiac hydatid cyst rupture from left heart causing ischemia and distal infarction by multiple emboli is a rare entity. We report such a case of a 24 year young Saudi male who was brought to Accident \& Emergency Department of Central Hospital, Arar, Saudi Arabia in unconsciousness condition. The patient was admitted with suspicion of over drinking or food poisoning.

In the emergency department the patient was resuscitated for loss of consciousness. The stomach lavage was done to minimize the systemic effect of food poisoning. A sample of the gastric lavage and a serum sample for the detection of alcohol were sent for confirmation of the provisional diagnosis of food poisoning and alcohol intoxication. The results of both the tests were reported to be negative, but the condition of the patient further deteriorated.

Patient was shifted to ICU for supportive measures. The internist on duty detected cold bluish discoloration of bilateral lower limbs without pulses. Vascular surgeon on duty was called upon and on color duplex a complete obstruction in abdominal aorta was detected at the renal level (Figure 1 and 2). In the meantime ECG/echocardiography was done and a mass was found in left ventricle of the heart. The contrast spiral CT-Scan from head to toe was also conducted to explore further findings.

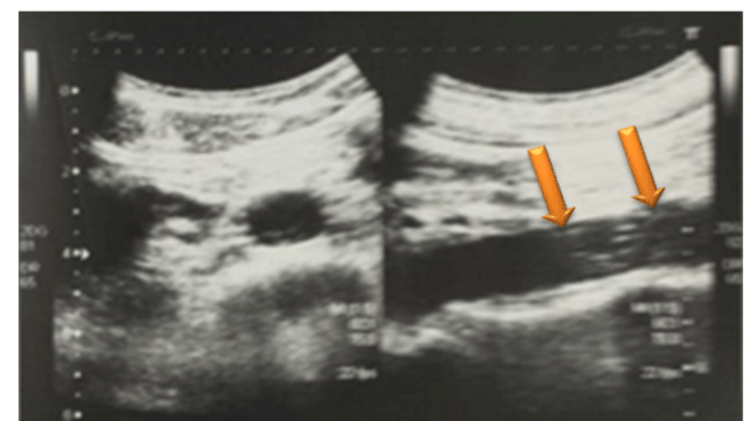

Figure 1: Grey scale ultrasound showing mixed echogenic floating membranes of hydatid cyst (Arrow) causing obstruction in distal abdominal aorta

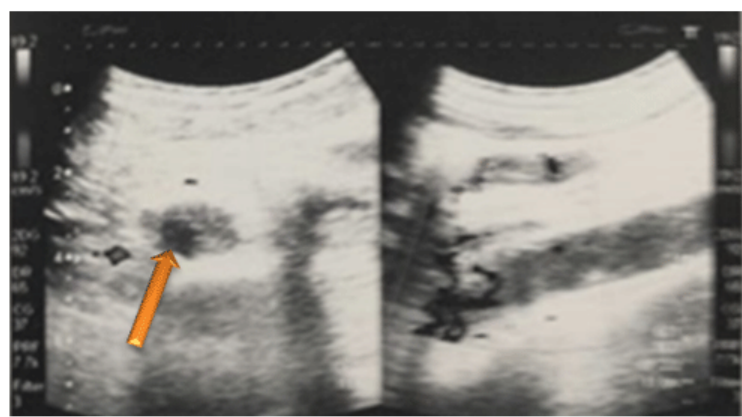

Figure 2: Color Duplex showing obstruction of abdominal aorta at infra renal level with minimal color flow in the center (Arrow)

CT-Scan confirmed large ruptured hydatid cyst in left lobe of liver (Figure 3), its communication and extension into left ventricle (Figure 3 and 4) and also demonstrated multiple emboli causing infarction at lower basal cerebelli, left occipital lobe (Figure 5), spleen (Figure 6), inferior pole left kidney (Figure 7) and ischemic changes in small gut (Figure 8). CT scan also showed displaced hydatid cyst membranes in lower abdominal aorta at infra renal level with extension into common iliac arteries resulting in partial obstruction (Figure 9).

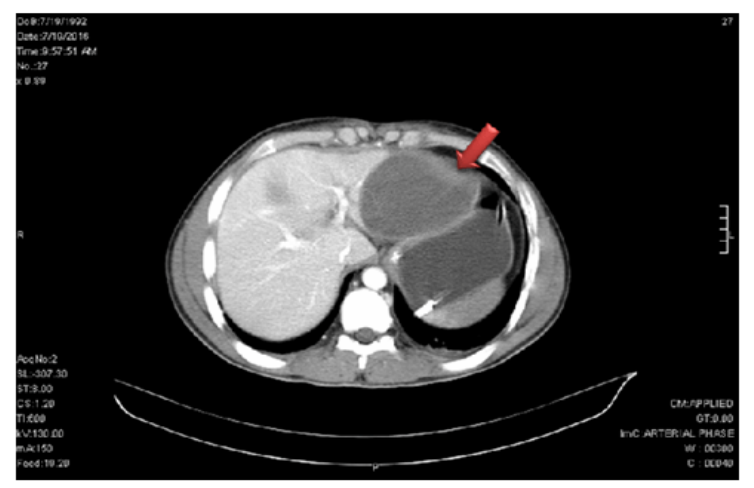

Figure 3: Contrast enhanced CT of abdomen showing a large Hydatid cyst in Left lobe of liver with ill-defined margins anteriorly suggesting rupture (Arrow)

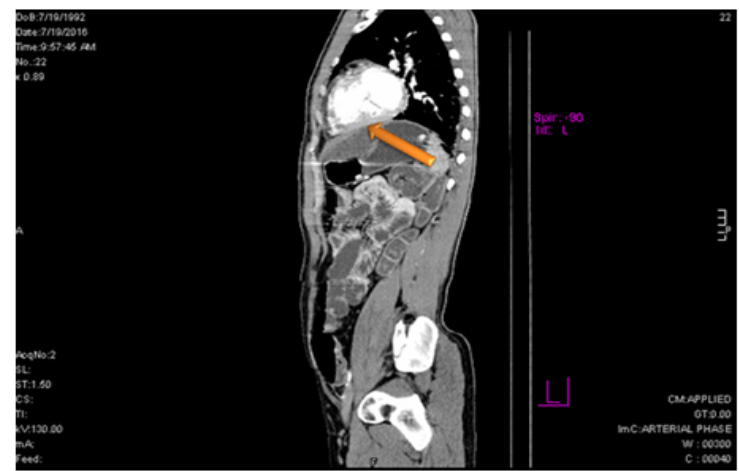

Figure 4: Contrast enhanced CT scan sagittal section, showing Hydatid cyst in left lobe of liver with extension to left ventricle

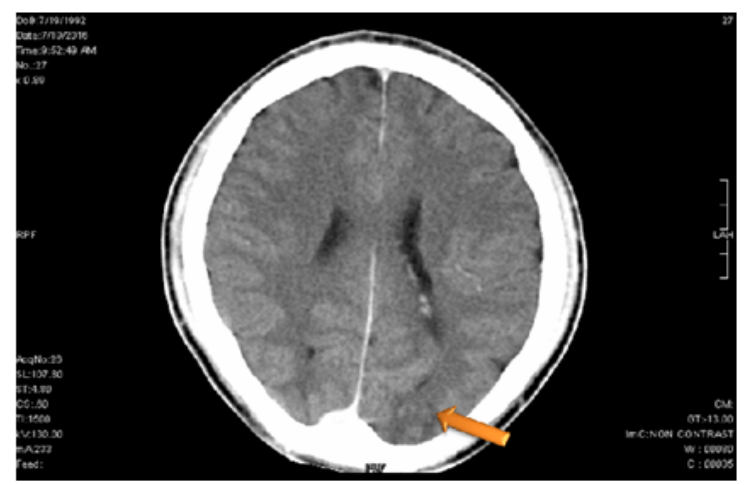

Figure 5: Non Contrast CT scan showing left occipital infarct (Arrow) 


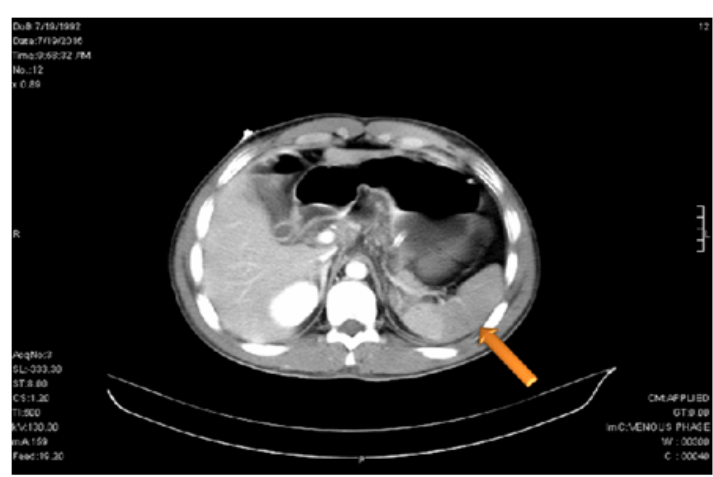

Figure 6: Contrast enhanced CT scan of Abdomen Axial section showing splenic infarct (Arrow)

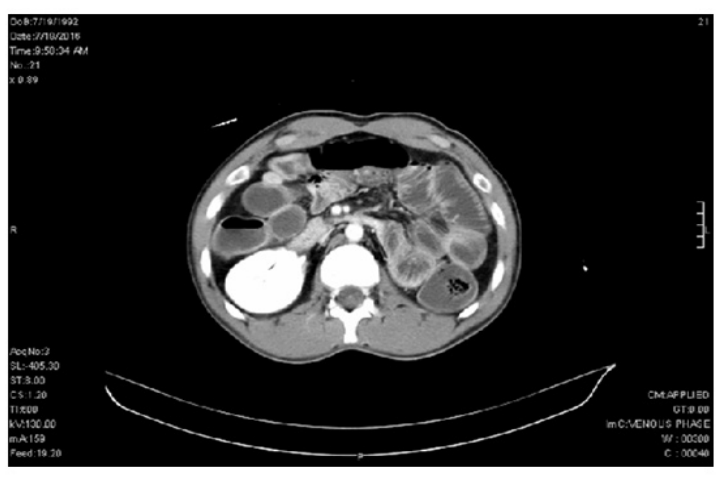

Figure 8: Contrast enhanced CT scan of Abdomen Axial section showing thickening of small bowel wall suggestive of ischemic changes

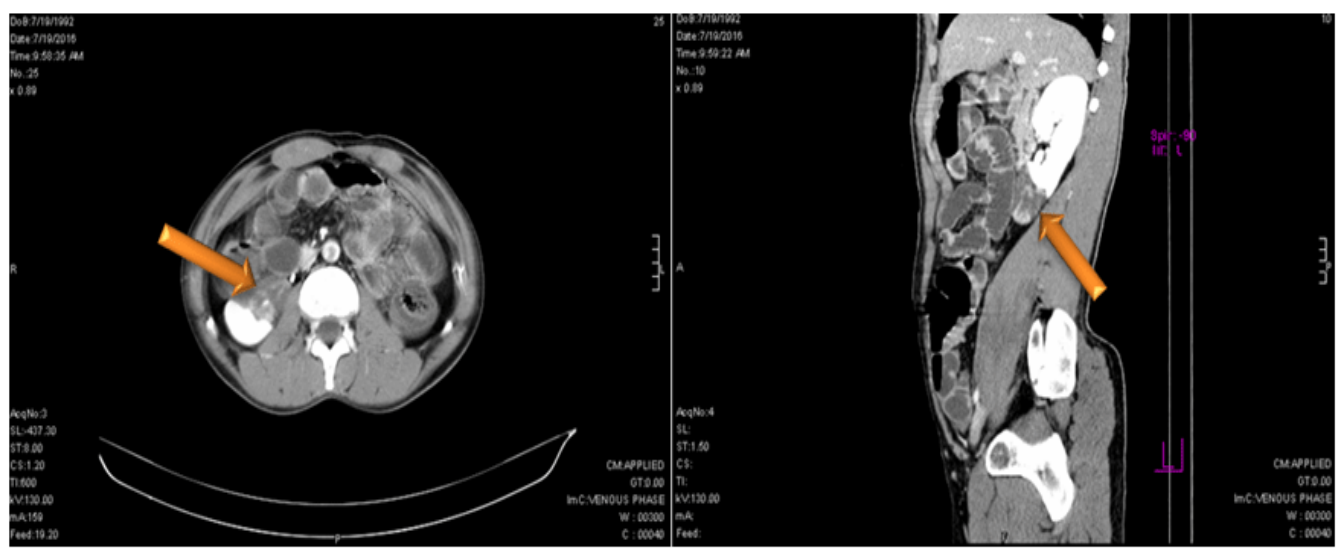

Figure 7: Contrast enhanced CT scan of Abdomen Axial and Sagittal section showing renal infarct at lower pole (Arrow)

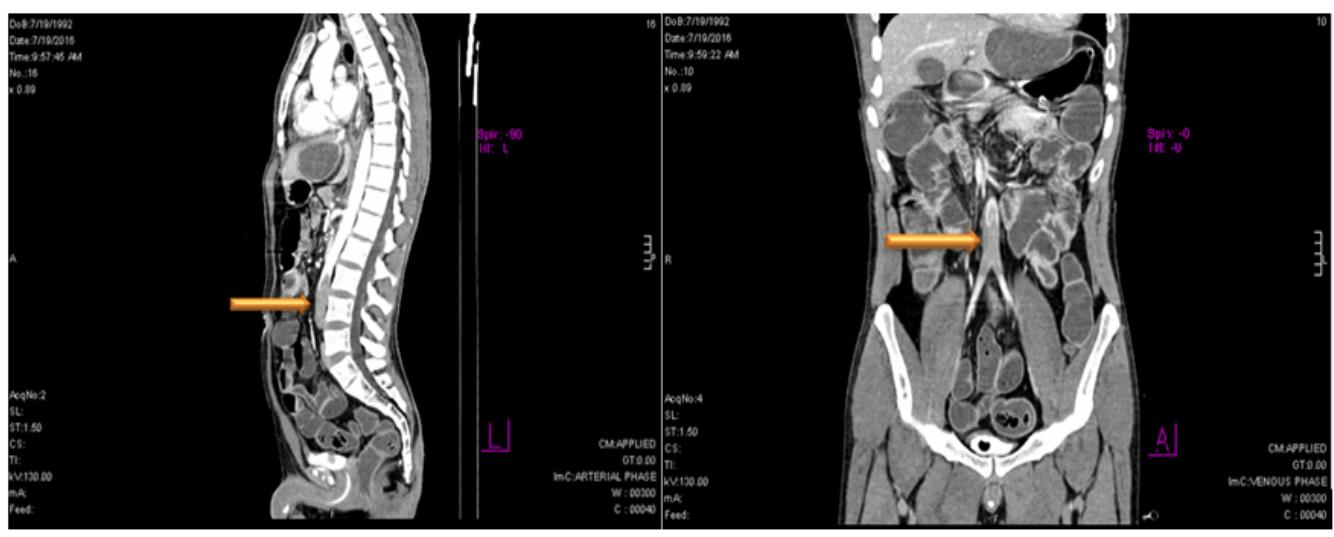

Figure 9: Contrast enhanced CT scan of Abdomen Coronal and Sagittal sections showing displaced hydatid cyst membranes (arrows) as filling defect in lower abdominal aorta causing aortic obstruction

With the collaborative surgery by cardiac and vascular surgeons the reperfusion of bilateral lower limbs was achieved by embolectomy with snare catheter. The fasciotomy of lower limbs was also performed to avoid the risk of reperfusion injury. The specimen from femoral embolectomy and a blood sample for
Casoni test were sent for the confirmation of hydated disease. The histopathology of the embolus revealed HC, while the Casoni test was negative.

In spite of all supportive measures patient expired 36 hours 
after surgery, possibly due to Systemic Inflammatory Response Syndrome (SIRS) and Multiple Organ Dysfunction Syndrome (MODS)

\section{Discussion}

As evident by this case report, admission of a comatose patient with suspicion of food poisoning or overdrinking obviously necessitated urgent resuscitation in hospital that upon deterioration was admitted to ICU. Workup in ICU revealed peripheral limb ischemia due to embolism of cardiac HC; similar cases have been reported in other studies [4, 6-10]. We did embolectomy but multiple infarcts and a bit delay in diagnoings underlying cause lead to SIRS, coagulopathy and ultimately resulted in the death of the patient.

Cardiac HC mostly involve left ventricle; it can erode heart in any adjacent pericardia vicinity sometimes causing local erosion into any cavity of heart leading to arrhythmias; similar picture was seen in our case where it eroded from liver to the left ventricle [10].

Surgical intervention is mandatory for relieving the intracardiac or intra-arterial obstruction as embolectomy which we did in our case; in addition surgery is needed for repairing the damaged cardiac walls and septa resulting from erosion of HC by open heart surgery; that was not noticed in our case [11]. Involvement of left and right atrium is nearly equal as epidemiological data suggested in few studies but we had left sided involvement in our case [4]. Sometimes cardiac tamponade resulting from pericardial rupture needs urgent decompression to avoid cardiac arrest [11,12]. Mitral valve involvement, which was not found in our case, can induce conduction disturbances, cardiac tamponade, or can be completely asymptomatic [13].

Cardiac hydatid cysts sometimes, with intra-cavity expansion, result in local ischemia to myocardium by pressure effect, sometimes eroding into adjacent areas [14]. Coronary artery bypass is mandatory for myocardial ischemia caused by hydatid cyst in order to save the life of the patient [15]. Rarely, cardiac hydatid cysts have been reported to manifest by causing ventricular tachycardia [16].

Moreover, the results of surgical treatment of heart HC are better than the conservative management only if vascular involvement is there $[11,17]$. On the other hand, there are major complications as reported by other studies, and also seen in our case, such as disseminated intravascular coagulation (DIC) ,systemic inflammatory response syndrome (SIRS) and multiple organ failure (MOF) leading to eventual death of the patient [18]

Keeping in consideration the pathological consequences like ischemia, surgical treatment such as embolectomy must be prompt; and should be followed by treatment with anthihelminthic chemotherapy such as mebendazole or albendazole with the aim to decrease the morbidity and mortality $[7,9-11,17,18]$.

\section{Conclusion}

As per our case report a young male was brought to a local hospital in coma with suspicion of over drinking or food poisoning. The patient was resuscitated and shifted to ICU where cold bluish discoloration of bilateral lower limbs without pulses was detected. Vascular surgeon on duty was called upon and on color duplex a complete obstruction in abdominal aorta was detected at the renal level. CT-Scan report confirmed ruptured HC in the left ventricle with multiple emboli and complete obstruction of lower abdominal aorta at renal level. The reperfusion of bilateral lower limbs was achieved by embolectomy. In spite of all supportive measures patient expired 36 hours after surgery, possibly due to Systemic Inflammatory Response Syndrome (SIRS) and Multiple Organ Dysfunction Syndrome (MODS). HC cyst is a rare entity leading to sudden embolism causing multiple ischemia and infarcts triggering a cascade to multiple organ failures if not treated in time.

\section{References}

1. Grozavu C, Ilias M, Pantile D. Multivisceral echinococcosis: concept, diagnosis, management. Chirurgia (Bucur). 2014;109(6):758-768.

2. Kelle S, Kohler U, Thouet T, Fleck E, Nagel E. Cardiac involvement of Echinococcus granulosus evaluated by multi-contrast CMR imaging. Int J Cardiol. 2009;131(2):e59-60.

3. Dursun M, Terzibasioglu E, Yilmaz R, Cekrezi B, Olgar S, Nisli K, et al. Cardiac hydatid disease: CT and MRI findings. AJR Am J Roentgenol. 2008;190(1):226-232.

4. Brunetti E, Kern P, Vuitton DA. Expert consensus for the diagnosis and treatment of cystic and alveolar echinococcosis in humans. Acta Trop. 2010;114(1):1-16. doi: 10.1016/j.actatropica.2009.11.001

5. Pedrosa I, Saiz A, Arrazola J, Ferreiros J, Pedrosa CS. Hydatid Disease: Radiologic and Pathologic Features and Complications. Radiographics. 2000;20(3):795-817.

6. Unlu Y, Ceviz M, Karaoglanoglu N, Becit N, Koçak H. Arterial embolism caused by a ruptured hydatid cyst in the heart: report of a case. Surg Today. 2002;32(11):989-991.

7. Ozer N, Aytemir K, Kuru G, Atalar E, Ozer Y, Ovunç K, et al. Hydatid cyst of the heart as a rare cause of embolization: report of 5 cases and review of published reports. J Am Soc Echocardiogr. 2001;14(4):299302 .

8. Shehatha J, Alward M, Saxena P, Konstantinov IE. Surgical management of cardiac hydatidosis. Tex Heart Inst J. 2009;36(1):72-73.

9. Rai N, Sarkar UN, Bhattacharya S, Sengupta G, Das D, Dutta S, et al. Hydatid cyst of the heart: An uncommon lesion. Ind J Thorac Cardiovasc Surg. 2009;25:211-213.

10. Besir Y, Gucu A, Surer S, Rodoplu O, Melek M, Tetik O. Giant cardiac hydatid cyst in the interventricular septum protruding to right ventricular epicardium. Indian Heart J. 2013;65(1):81-83. doi: 10.1016/j.ihj.2012.12.014

11. Tuncer E, Tas SG, Mataraci I, Tuncer A, Donmez AA, Aksut M, et al. Surgical treatment of cardiac hydatid disease in 13 patients. Tex Heart Inst J. 2010;37(2):189-193.

12. Elkarimi S, Ouldelgadia N, Gacem H, Zouizra Z, Boumzebra D, Blelaabidia B, et al. Tamponade reveals an intra-pericardial hydatid cyst-a case report. Ann Cardiol Angeiol (Paris). 2014;63(4):267-270. doi: 10.1016/j.ancard.2012.01.003

13. Aksakal E, Degirmenci H, Bakirciu EM. PP-139 A Case of Mitral Valve Involvement by Hydatid Cyst Disease. International Journal of Cardiology. 2010;140:S80.

14. Kaplan M, Demirtas M, Cimen S, Ozler A. Cardiac hydatid cysts with intracavitary expansion. Ann Thorac Surg. 2001;71(5):1587-1590. 
15. Henaine R, Mathevet JL, Rouviere H, Di-Filippo S, Cannesson M, Obadia $\mathrm{JF}$, et al. Coronary artery bypass in myocardial ischemia of the young due to hydatid cyst. Journal of cardiac surgery. 2008;23(5):573-575.

16. Elhadj ZI, Boukhris M, Kammoun I, Halima AB, Addad F, Kachboura S Cardiac hydatid cyst revealed by ventricular tachycardia. J Saudi Heart Assoc. 2014;26(1):47-50. doi: 10.1016/j.jsha.2013.08.001
17. Birincioglu CL, Bardakci H, Kucuker SA, Ulus AT, Arda K, Yamak B, et al. A clinical dilemma: cardiac and pericardiac echinococcosis. Ann Thorac Surg. 1999;68(4):1290-1294.

18. Di Bello R, Menendez H. Intracardiac rupture of hydatid cysts of the heart. A study based on three personal observations and 101 cases in the world literature. Circulation. 1963;27(3):366-374. 